\title{
A systematic review of the effect of physical exercise on cognition in stroke and traumatic brain injury patients
}

\author{
Ines Vanderbeken ${ }^{\mathrm{a}, *}$ and Eric Kerckhofs ${ }^{\mathrm{b}, \mathrm{c}}$ \\ ${ }^{a}$ Rehabilitation Center Acquired Brain Injuries Turnhout, Turnhout, Belgium \\ ${ }^{\mathrm{b}}$ Center for Neurosciences, Vrije Universiteit Brussels, Brussels, Belgium \\ ${ }^{\mathrm{c}}$ Psychology and Educational Sciences, Vrije Universiteit Brussels, Brussels, Belgium
}

\begin{abstract}
.
OBJECTIVE: To determine whether physical exercise enhances cognition following TBI or stroke.

DATA SOURCES: Studies were identified through searches of PubMed, ScienceDirect and the reference lists of papers that were included for full-text evaluation. Medical subject headings from three concepts, i.e. brain injury, physical exercise and cognition, were used to incorporate related search terms.

STUDY SELECTION: Included were all trials published in English that assessed cognition before and after an exercise intervention in human adults with TBI or stroke. Nine randomized and two non-randomized controlled trials, as well as three single group pre-post studies were included.

DATA EXTRACTION: Relevant data concerning the methods and results of the included studies were extracted. Methodological quality of the RCT's was evaluated using the PEDro scale. Non-randomized trials were assessed using the Downs and Black checklist.

DATA SYNTHESIS: The included trials were generally of medium methodological quality, though often plagued with issues of internal and external validity. The studies exhibited great heterogeneity, rendering a meta-analysis infeasible.

CONCLUSIONS: Though well-designed studies are still needed, the preponderance of evidence suggests a positive effect of physical exercise on global cognitive functioning, especially in the chronic stages of a brain injury. Time after injury as well as the duration of the exercise program are mediating factors.
\end{abstract}

Keywords: TBI, stroke, brain injuries, exercise, cognition
Abbreviations
CCT
clinical controlled trial
CT clinical trial
ABI
acquired brain injury (of any cause)
CVA
cerebrovascular accident
AT aerobic training
D\&B
Downs and Black
*Address for correspondence: Ines Vanderbeken, Rehabilita-
FIM-cog Functional Independence Measure, tion Center Acquired Brain Injuries Turnhout, Korte Begijnen- straat 18, 2300 Turnhout, Belgium. Tel.: +32 144436 47/+32 4951096 27; E-mails: ines.vanderbeken@kmsl.be, vanderbeken. ines@gmail.com.
Cognition Subscale
MMSE Mini Mental State Examination
MoCA Montreal Cognitive Assessment
PEDro Physiotherapy Evidence Database 


$\begin{array}{ll}\text { RCT } & \text { randomized controlled trial } \\ \text { RT } & \text { resistance training } \\ \text { TBI } & \text { traumatic brain injury } \\ \text { TMT } & \text { Trail Making Test }\end{array}$

\section{Introduction}

The idea that physical exercise yields positive effects on the body can be regarded as common knowledge. Not only has it been shown to be a prevention tool against all-cause mortality (Lee \& Skerrett, 2001), it can also be used as a secondary disease treatment for chronic illnesses (Durstine, Gordon, Wang, \& Luo, 2013). As such, it can attenuate side effects and might even increase survival rates when facing a life-threatening illness such as breast cancer (Casla et al., 2014; Kim, Choi, \& Jeong, 2013). The idea that physical exercise can influence our brain is more recent, as scientific research into its plasticity only started around the 1940's (Hebb, 1949). During the last few decades, a large body of nonhuman animal research and a growing number of studies on humans show structural differences in the brain such as increases in growth factors (Szuhany, Bugatti, \& Otto, 2015), higher levels of neurogenesis (van Praag, 2008), longer dendrites with a more complex morphology (Redila \& Christie, 2006), enhanced long-term potentiation (van Praag, Christie, Sejnowski, \& Gage, 1999), alterations in neurotransmitter systems (Meeusen, 2005), changes in cerebral blood flow (Ogoh \& Ainslie, 2009), greater grey matter volume (Erickson, Leckie, \& Weinstein, 2014), increased angiogenesis (Schmidt, Endres, Dimeo, \& Jungehulsing, 2013) and higher levels of synaptogenesis (Ambroginia et al., 2013). These structural changes go hand in hand with changes in everyday living such as positive effects on mood (Duman, 2005), improved sleep quality (Yang, Ho, Chen, \& Chien, 2012) and enhanced cognitive function (Hötting \& Röder, 2013).

Certain subgroups such as the elderly and children have been the focal point of interest due to the 'age dependent hypothesis' (Hötting \& Röder, 2013). The idea is that the brain will be more susceptible to alterations brought about by physical exercise when in periods of change, whether it be growth or decline. But what about when the brain is undergoing changes due to an acquired brain injury (ABI)?
The number of people suffering from a brain injury is staggering. In 2010, there were approximately 2.5 million emergency department visits, hospitalizations and deaths in the United States where traumatic brain injury (TBI) was a diagnosis (Centers for Disease Control and Prevention [CDC], 2014). Moreover, there are around 6.6 million Americans over the age of twenty that have suffered a stroke, and its yearly incidence rate is estimated at around 800,000 persons (Mozaffarian et al., 2015). As lifesaving technology continues to make headway, one can only presume that the number of survivors will grow. Ergo, even more people will find themselves with disability due to the motor, cognitive, emotional and behavioral consequences of a brain injury. The cost of these disabilities is great, not only for directly affected individuals and their families, but also for society as a whole (Olesen et al., 2015). Therefore, it stands to reason that any method that could help alleviate symptoms is worth exploring. We have chosen to focus specifically on cognition, as cognitive disabilities and perceived cognitive self-efficacy have been shown to be an important predictor of quality of life in stroke (Cumming, Brodtmann, Darby, \& Bernhardt, 2014) and TBI victims (Cicerone \& Azulay, 2007).

Several reviews have already shown that there are cognitive benefits to exercising in diverse neurological populations (Heyn, Abreu, \& Ottenbacher, 2004; McDonnell, Smith, \& Mackintosh, 2011). There have also been some reviews on this subject about TBI and/or stroke victims (Cumming, Tyedin, Churilov, Morris, \& Bernhardt, 2012; Devine \& Zafonte, 2009; Fogelman \& Zafonte, 2012). In light of recent scientific activity, i.e. four of the fourteen included studies are dated from 2012 or later, we believe that an update of the literature is in order.

This review is meant to provide an overview of the research that is currently available on the effect of physical exercise on cognition in TBI and stroke patients. In this regard, we will attempt to determine which cognitive functions are malleable through physical exercise and which are not. In addition, we will search for information to discover if these changes are of a transitory or more permanent nature. Furthermore, we will try to shed light on the upper and lower limits of the amount of physical exercise needed to support cognitive changes, as well as on the types of physical exercise that induce these changes. We will conclude this review with suggestions for further research. 


\section{Methods}

\subsection{Criteria for considering studies for this review}

The PICOS method (Liberati et al., 2009) was implemented to delineate the five components of our literature review. $\mathrm{P}$ (patient)= human adults diagnosed with a stroke or TBI. I (intervention) $=$ all types of physical exercise, defined as 'any form of musculoskeletal movement that raises energy consumption markedly over resting energy expenditure'. $\mathrm{C}($ comparison $)=\mathrm{a}$ second type of physical therapy intervention, interventions that did not involve physical exercise or no intervention. We excluded studies where physical exercise was only part of the intervention, such as the study by Rand, Eng, Liu-Ambrose, and Tawashy (2010), since determining the exact role of physical exercise would not be possible in such studies. O (outcome) = scores on neuropsychological tests or clinical observation scales measuring cognition (also referred to as cognitive function), administered by a researcher or clinician. Cognition was defined as: 'the basic ability of the brain to process, store, retrieve and manipulate information to solve problems' (Prigatano, 1986). S (study design $)=$ randomized controlled trials $($ RCT $)$, clinical controlled trials (CCT) and clinical trials (CT). The latter two are non-randomized trials that only differ on the inclusion of a control group. Case studies, reports published in conference proceedings and retrospective studies were not included. The language of the articles was restricted to English. Date limits were not applied. Table 1 provides an overview of the inclusion and exclusion criteria.

\subsection{Search strategies}

The included papers were obtained through searches of the electronic databases PubMed and ScienceDirect. For PubMed, we used MeSH terms to determine the optimal combination of key words. Non-human animal studies were set to be automatically excluded. For ScienceDirect, we used the option to only include papers if our keywords appeared in the title, abstract or key words. Since it was not possible to automatically exclude animal trials in this search engine, this was done through hand search (see Table 1 for search details). We also searched the reference lists of articles included for full-text evaluation to identify previously missed relevant studies and checked suggested related articles for

Table 1

Inclusion and exclusion criteria and search details

\begin{tabular}{|c|c|c|}
\hline & Inclusion criteria & Exclusion criteria \\
\hline Participants & $\begin{array}{l}\geq 18 \text { years } \\
\text { Diagnosis of stroke (ICD-10: I60-I63) or TBI } \\
\quad(\text { ICD-10: S06.2-S06.9) }\end{array}$ & Non-human subjects \\
\hline Intervention & $\begin{array}{l}\text { Physical exercise interventions (e.g. aerobic training, } \\
\text { resistive training, Bobath techniques...) }\end{array}$ & Physical exercise as only part of the intervention \\
\hline Comparison & $\begin{array}{l}\text { A second physical exercise intervention, other } \\
\text { interventions (e.g. relaxation therapy, passive } \\
\text { mobilization of arms/legs, stretching ...) } \\
\text { or no intervention }\end{array}$ & \\
\hline Outcome & $\begin{array}{l}\text { Separate measures for neurocognitive function, } \\
\text { administered by a researcher or clinician }\end{array}$ & $\begin{array}{l}\text { Questionnaires (self- or proxy-) as the only } \\
\text { measure of cognitive function or no cognitive } \\
\text { measures }\end{array}$ \\
\hline Study design & $\begin{array}{l}\text { Randomized controlled trials, clinical controlled } \\
\text { trials and clinical trials }\end{array}$ & $\begin{array}{l}\text { Reports of conference proceedings and case } \\
\text { reports }\end{array}$ \\
\hline Type of articles & Journal articles without date limits & Non-English publications \\
\hline Search details PubMed & \multicolumn{2}{|c|}{ 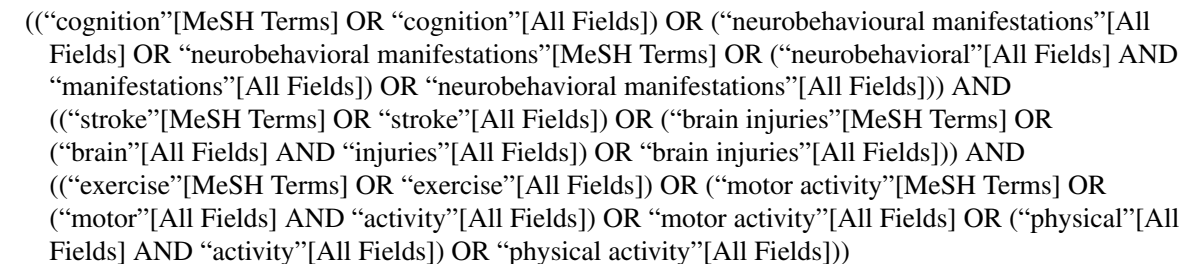 } \\
\hline Search details ScienceDirect & \multicolumn{2}{|c|}{$\begin{array}{l}\text { TITLE-ABSTR-KEY((Stroke OR ABI OR brain injury OR TBI OR CVA) AND (physical activity OR } \\
\text { exercise) AND (cognition OR cognitive OR brain)) [All Sources(- All Sciences -)] }\end{array}$} \\
\hline
\end{tabular}


Table 2

Levels of evidence

\begin{tabular}{|c|c|c|}
\hline Level & Research design & Description \\
\hline 1 & RCT & $\begin{array}{l}\text { RCT, PEDro score } \geq 6 \text {. Includes within-subjects comparison with randomized conditions and cross-over } \\
\text { designs. }\end{array}$ \\
\hline \multirow[t]{2}{*}{2} & RCT & RCT, PEDro score <6. Prospective controlled trial. Prospective controlled trial (not randomized). \\
\hline & Cohort & Prospective longitudinal study using at least 2 similar groups with one exposed to a particular condition. \\
\hline 3 & Case control & A retrospective study comparing conditions, including historical controls. \\
\hline \multirow[t]{3}{*}{4} & Pre-post & A prospective trial with a baseline measure, intervention, and a post-test using a single group of subjects. \\
\hline & Post-test & $\begin{array}{l}\text { A prospective post-test with two or more groups - intervention, then post-test (no pre-test or baseline } \\
\text { measurement) using a single group of subjects. }\end{array}$ \\
\hline & Case Series & A retrospective study usually collecting variables from a chart review. \\
\hline \multirow[t]{3}{*}{5} & Observational & Study using cross-sectional analysis to interpret relations. \\
\hline & Clinical Consensus & Expert opinion without explicit critical appraisal, or based on physiology, biomechanics or "first principles". \\
\hline & Case Report & Pre-post or case series involving one subject. \\
\hline
\end{tabular}

Reprinted, with permission, from Eng JJ, Teasell RW, Miller WC, et al. Spinal cord injury rehabilitation evidence: Method of the SCIRE systemic review. Top Spinal Cord Inj Rehabil. 2007;13(1):1-10. Copyright 2007 Thomas Land Publishers, Inc.

relevance. While the searches were not limited to original research, reviews are not discussed in the results. They were carefully screened for relevant articles and mentioned in the discussion when deemed fitting. An a priori search protocol was developed and used and the final search for both search engines was performed on February 28 of 2015. For optimal reporting, we used the PRISMA Statement (Liberati, 2009).

\subsection{Data collection and analysis}

\subsubsection{Study selection}

The titles of articles generated by the previously defined search actions were examined by the author IV to discard clearly irrelevant articles. Abstracts of the remaining publications were scrutinized for eligibility by two independent assessors (i.e. the author IV and physiotherapist $\mathrm{NH}$ ), using the criteria described above. Full-texts were acquired for all articles that were either eligible for inclusion or for which eligibility was unclear and were read by both assessors. If disagreement arose on whether or not to include a publication, a third independent party (author EK) was asked to settle the matter.

\subsubsection{Assessment of methodological quality}

All eligible trials were judged on methodological rigor by means of the PEDro (Physiotherapy Evidence Database) scale (www.pedro.org.au) or the Downs and Black (D\&B) checklist (Downs \& Black, 1998), depending on study design. Studies not meeting the threshold for a fair quality rating were excluded from our analysis. In addition, we determined the levels of evidence (Table 2) using a table designed for spinal cord injury rehabilitation, which was easily applicable to stroke rehabilitation literature (Eng et al., 2007).

\subsubsection{Randomized controlled trials-PEDro scale.}

The most commonly used scale to assess RCT's in the entire field of health care is the Jadad scale (Jadad et al., 1996). However, its validity for physical therapy studies has not been supported (Olivo et al., 2008). It is a scale comprised of three criteria, namely randomization, double blinding and withdrawals/dropouts. The PEDro scale encompasses these three criteria and has been shown to be a more comprehensive measure of methodological quality when reviewing stroke rehabilitation literature (Bhogal, Teasell, \& Foley, 2005). Furthermore, it is one of the most commonly used scales in physical therapy research (Olivo et al., 2008). It consists of 11 items and except for item one, pertaining to external validity, each satisfied internal validity criterion leads to an additional point, thus leading to a maximal combined score of 10 with: $9-10=$ excellent, $6-8=$ good, $4-5=$ fair, below $4=$ poor. The scores for each RCT were retrieved on the PEDro website (Table 3).

\subsubsection{Non-randomized trials - Downs and Black} checklist. Research evaluating the quality of the PEDro scale has focused solely on its application for randomized controlled trials (Bhogal et al., 2005; Foley, Bhogal, Teasell, Bureau, \& Speechley, 2006; Maher, Sherrington, Herbert, Moseley, \& Elkins, 2003). For this reason, we chose to use a different tool to assess the quality of our non-randomized studies, i.e. the Downs and Black checklist (Downs \& Black, 1998). This checklist may not be used as commonly 
Table 3

Scores for methodological quality, sample size and the levels of evidence

\begin{tabular}{|c|c|c|c|c|}
\hline & Study/year & PEDro score & Downs and Black score & $\overline{\text { Level of evidence }}$ \\
\hline \multicolumn{5}{|l|}{$\mathrm{ABI}$} \\
\hline & Bateman et al., 2001 & 7/10 (good) & - & Level 1 \\
\hline \multicolumn{5}{|c|}{ 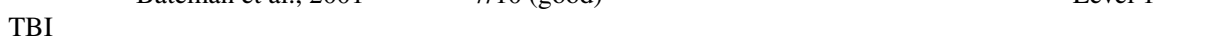 } \\
\hline & McMillan et al., 2002 & 6/10 (good) & - & Level 1 \\
\hline & Grealy et al., 1999 & $1 / 10$ (poor) & - & Level 2 \\
\hline & Chin et al., 2014 & - & 16/28 (fair) & Level 4 \\
\hline \multicolumn{5}{|l|}{ CVA } \\
\hline & Moore et al., 2014 & 7/10 (good) & - & Level 1 \\
\hline & Nilsson et al., 2001 & $7 / 10$ (good) & - & Level 1 \\
\hline & Fang et al., 2003 & $6 / 10$ (good) & - & Level 1 \\
\hline & Quaney et al., 2009 & $6 / 10$ (good) & - & Level 1 \\
\hline & Studenski et al., 2005 & $6 / 10$ (good) & - & Level 1 \\
\hline & Ploughman et al., 2008 & $5 / 10$ (fair) & - & Level 2 \\
\hline & Marzolini et al., 2012 & - & 18/28 (fair) & Level 4 \\
\hline & Kluding et al., 2011 & - & 15/28 (fair) & Level 4 \\
\hline & El-Tamawy et al., 2014 & - & 15/28 (fair) & Level 2 \\
\hline & Pyöriä et al., 2007 & - & $15 / 28$ (fair) & Level 2 \\
\hline
\end{tabular}

Table 4

Scores on the subscales of the modified Downs and Black checklist

\begin{tabular}{llcccc}
\hline & Study/year & Reporting & External validity & Bias & Confounding \\
\hline TBI & & & & & \\
& Chin et al., 2014 & $10 / 11$ & $0 / 3$ & $4 / 7$ & $2 / 7$ \\
CVA & & & & \\
& & & & $4 / 7$ & $1 / 7$ \\
& El-Tamawy et al., 2014 & $9 / 11$ & $0 / 3$ & $4 / 7$ & $1 / 7$ \\
& Kluding et al., 2011 & $10 / 11$ & $1 / 3$ & $4 / 7$ & $3 / 7$ \\
& Marzolini et al., 2012 & $10 / 11$ & $3 / 3$ & $4 / 7$ & $1 / 7$ \\
\hline
\end{tabular}

in physical therapy research as the PEDro scale, it has however been found to be the best in its category compared to 18 other instruments for evaluating non-randomized trials (Saunders, Soomro, Buckingham, Jamtvedt, \& Raina, 2003). Moreover, the PEDro scale and the D\&B tool correlate moderately highly (Aubut, Marshall, Bayley, \& Teasell, 2013). The D\&B checklist consists of five subscales, namely Reporting, External validity, Internal validity (bias), Internal validity (confounding) and Power. Since the tool has a final question with a range from 0 to 5 which makes it difficult to score accurately, we used the revised version by Eng et al. (2007). They reduced the final question to a yes or no answer, resulting in a maximal total score of 28 . Quality categories were defined as 26-28=excellent, 20-25=good, 15-19=fair and below $14=$ poor (Benjamin, van de Water, \& Peiris, 2014; Hooper, Jutai, Strong, \& Russsel-Minda, 2008). Scores were calculated by the author and physiotherapist NH (Tables 3 and 4). Higher scores represent a higher methodological quality. Again, if disagreement arose, the procedure described above was used.

\subsection{Data extraction}

Relevant data concerning the methods and results of the included studies were extracted, including study design, tools used for cognitive assessment, details with regard to the population, type and duration of interventions, neurocognitive outcomes post-intervention etc. (Table 5).

\section{Results}

\subsection{Identification and selection of studies}

The search strategy (Fig. 1) rendered a total of 162 hits in ScienceDirect and 772 results in PubMed. After eliminating doubles and clearly irrelevant articles by title and/or abstract, 27 publications were eligible for this review. Of these 27 articles, 12 were deemed relevant after reading the full-text; 9 were clinical trials, 3 were reviews. By hand searching the references of these 27 articles, an additional 5 relevant trials emerged, resulting in a final total of 14 


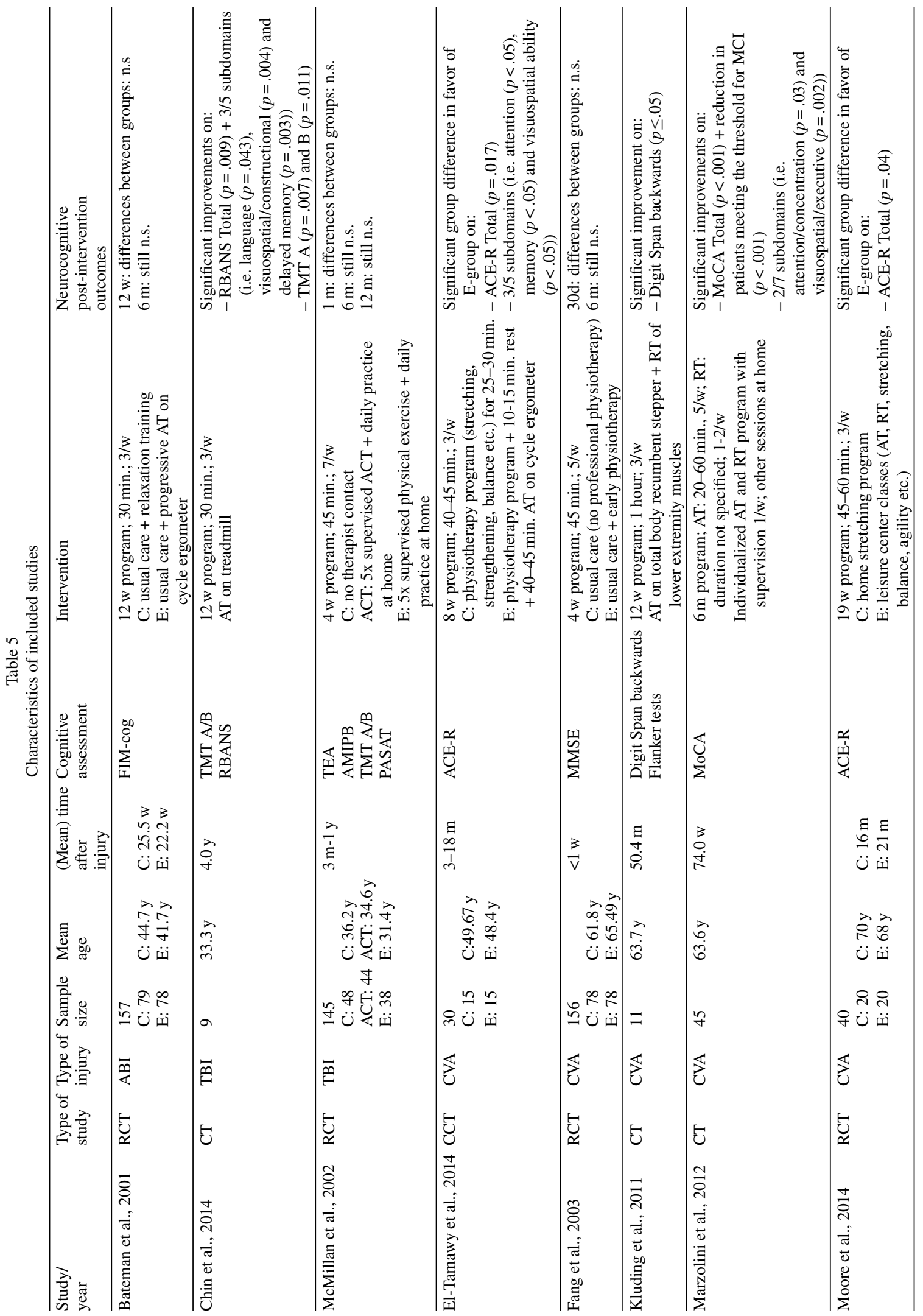




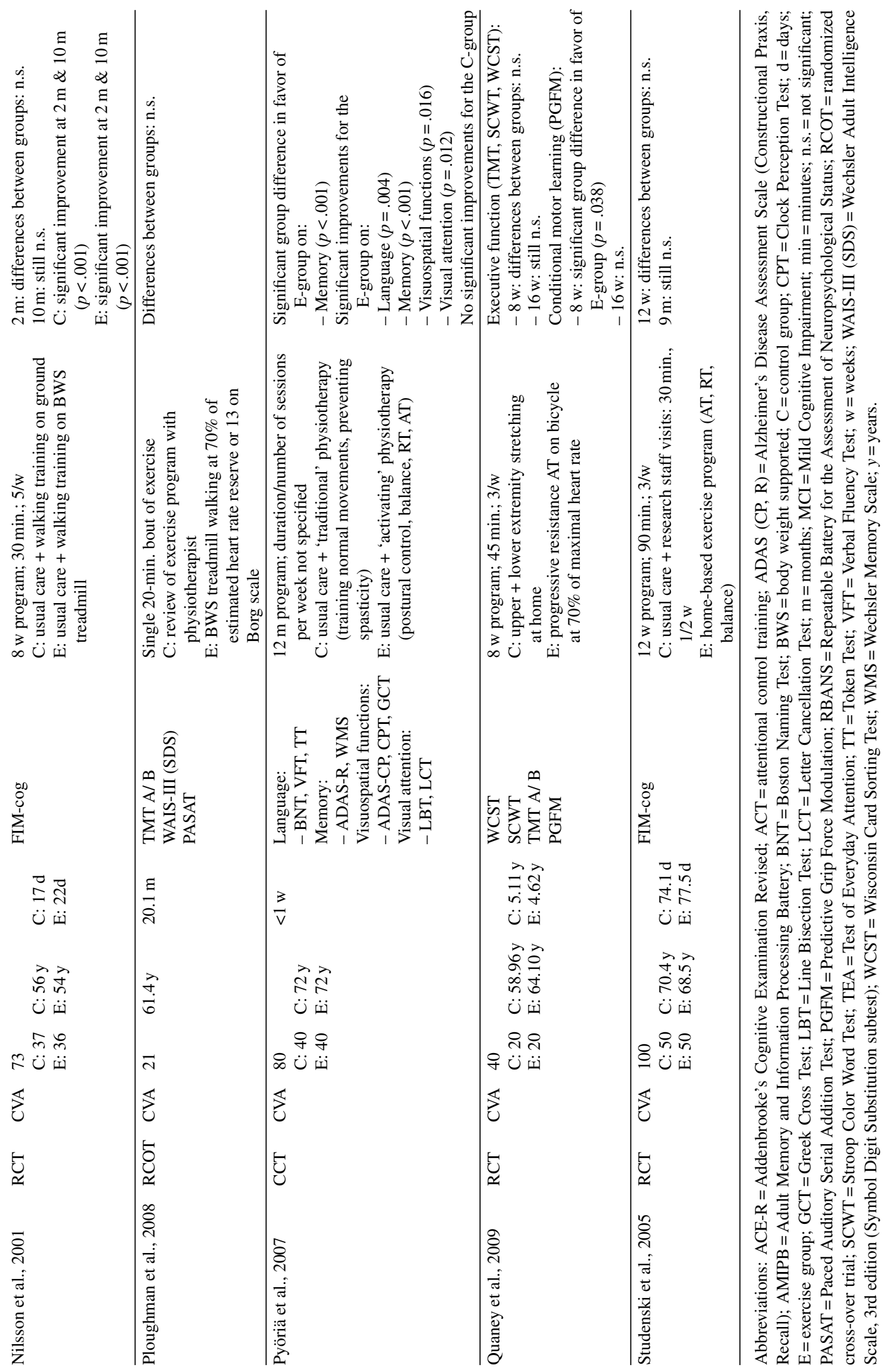




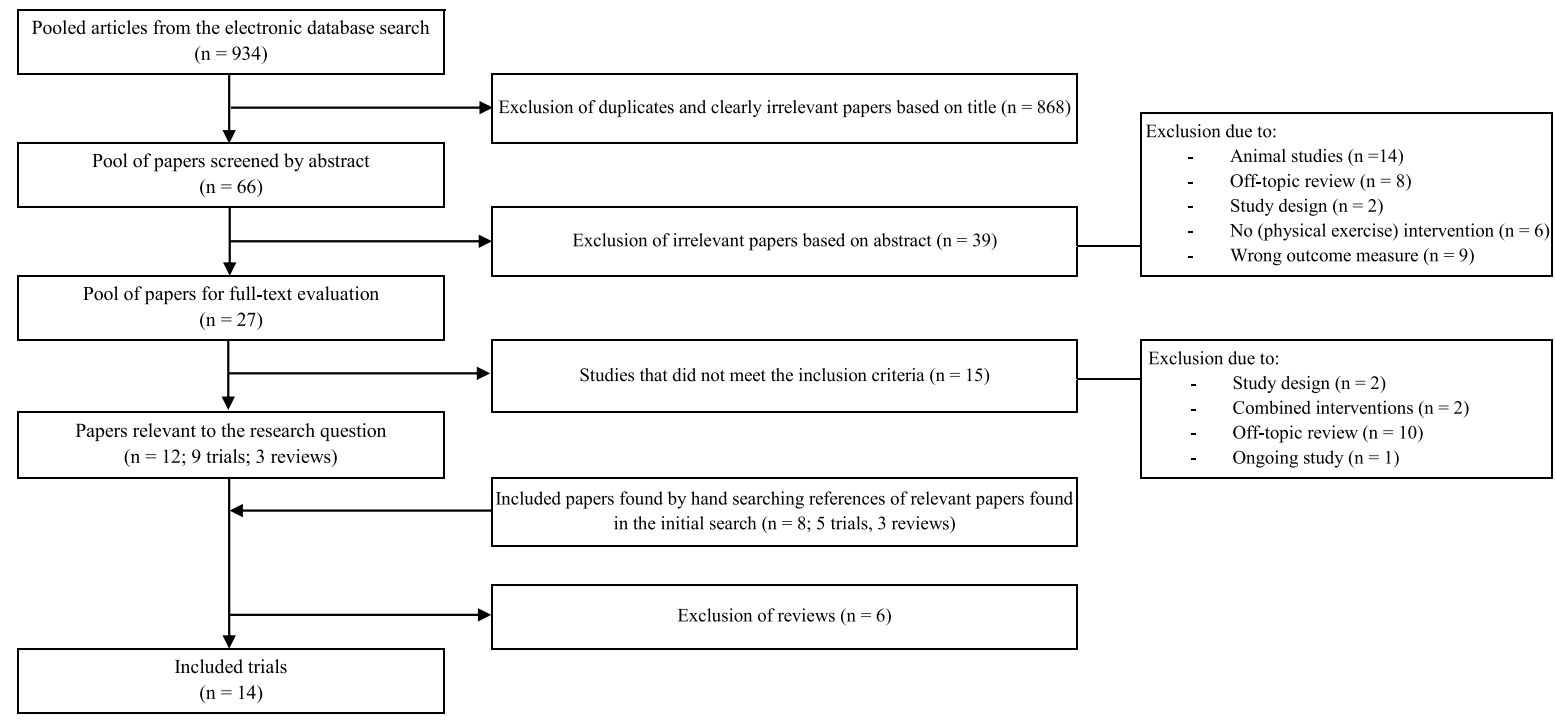

Fig. 1. Identification and selection of studies.

included studies dated from 1999 to 2014. Due to the low number of RCT's investigating the effect of physical exercise on cognition (i.e. 9), CCT's and CT's were also taken into consideration. However, more weight was attributed to the conclusions of studies with greater methodological rigor.

\subsection{Quality assessment}

The RCT's are of good quality according to PEDro standards, with two exceptions (Table 3 ). The first is a study by Ploughman, McCarthy, Bossé, Sullivan, and Corbett (2008) with a 'fair' quality rating, and the second is a study by Grealy, Johnson, and Rushton (1999) which is markedly methodologically flawed (i.e. PEDro score: 1/10). For this reason, the latter study was excluded from analysis and will not be discussed further.

Three of the non-randomized trials generated a score of 15 out of 28 , while the other two received slightly higher scores of 16 and 18 (Table 3). This means that all studies received a 'fair' quality rating. More specifically, they were mostly well reported, allowing the reader to make an unbiased assessment of the results, though there were issues with validity. Namely, all five trials showed low external validity and a relatively high risk of the presence of a selection bias (Table 4).

\subsection{Exercise and neurocognition following ABI}

Due to the heterogeneity of the subject characteristics, interventions and outcome measures employed in these studies, a meaningful meta-analysis was not feasible. A summary of the results is presented in Table 5.

\subsubsection{Duration and intensity of exercise programs}

3.3.1.1. A single bout of exercise. Only one article measured whether a single bout of exercise could enhance cognitive function (Ploughman et al., 2008). This within-subject cross-over study did not find beneficial effects of exercise on cognition in stroke victims after a 20-minute bout of aerobic training (AT). Similar studies with TBI patients have yet to be published. Obviously more research is needed before definite conclusions regarding the effectiveness of single bouts of exercise in our population can be made.

3.3.1.2. Recurring exercise. Recurring exercise programs lasted four weeks to one year with patients exercising three times a week to daily and ranging from 30 to 90 minutes per session. Session duration and the number of times a patient exercised a week did not determine outcome. However, the duration of the exercise program did appear to influence results. We found that the two shortest programs (i.e. of four weeks) did not elicit cognitive benefits (Fang et al., 2003; McMillian, Robertson, Brock, \& Chorlton, 2002). Contrarily, almost all studies with programs of eight weeks or more - including studies of both good and fair methodological quality - found cognitive gains (Chin, Keyser, Dsurney, \& Chan, 2015; El-Tamawy, Abd-Allah, Ahmed Darwish, \& 
Khalifa, 2014; Kluding, Tseng, \& Billinger, 2011; Marzolini, Oh, Mcllroy, \& Brooks, 2013; Moore et al., 2015; Nilsson et al., 2001; Pyöriä et al., 2007; Quaney et al., 2009).

\subsubsection{Type of exercise}

Only two studies directly compare exercise conditions (Nilsson et al., 2001; Pyöriä et al., 2007). The first is a level 1 study of good quality which compares AT on a treadmill with AT on the ground (Nilsson et al., 2001). The results show no significant differences between these exercise methods. The second is a level 2 study of fair quality which compares a group receiving traditional physiotherapy (i.e. Bobath therapy) with an activating therapy group (i.e. based on principles of the Motor Relearning Program) (Pyöriä et al., 2007). Not surprisingly, both groups improved significantly on physical functioning capacity, but only activating therapy - which challenges patients more on a cognitive level - significantly improved memory, language, visuospatial abilities and visual attention. However, a significant group difference was only found for memory.

3.3.2.1. Aerobic training. Several studies used recurring aerobic training for the exercise intervention (Bateman et al., 2001; Chin et al., 2015; El-Tamawy et al., 2014; Nilsson et al., 2001; Quaney et al., 2009). A level 2 (El-Tamawy et al., 2014) and level 4 (Chin et al., 2015) study of fair quality and two level 1 studies (Nilsson et al., 2001; Quaney et al., 2009) of good quality all found significant effects, thus leading us to conclude that AT can positively influence cognition in our population.

3.3.2.2. Aerobic and resistance training. Two publications used AT and combined it with resistance training (RT) (Kluding et al., 2011; Marzolini et al., 2013). Both are level 4 studies of fair methodological quality. Their patient groups improved significantly on several measures of cognitive functioning after a relatively intensive exercise program that lasted three (Kluding et al., 2011) to six (Marzolini et al., 2013) months. Given the quality of these two studies, we merely consider this an indication that AT and RT may be an effective combination for improving cognition.

3.3.2.3. Mixed training programs. Two studies compare a mixed training program to usual care (Fang et al., 2003; Studenski et al., 2005), while one compares it to a home stretching program (Moore et al., 2015).
All are level 1 studies of good quality, however they do not all come to similar conclusions. The study with the highest quality score found significant differences in global cognitive functioning in favor of its exercise group (Moore et al., 2015). In contrast, the other two studies were not able to find any neurocognitive benefit to exercising (Fang et al., 2003; Studenski et al., 2005). The many differences between these studies in treatment length and type, outcome measure, time after injury and whether or not exercise is performed in group may all account for these diverging results.

\subsubsection{Neurocognitive function}

3.3.3.1. Global neurocognitive functioning. The majority of evidence suggests that recurring exercise can benefit global cognitive functioning. This is evidenced by two level 4 studies (Chin et al., 2015; Marzolini et al., 2013) and one level 2 study (ElTamawy et al., 2014) of fair quality together with one level 1 study (Moore et al., 2015) of good quality. All used a screening tool and found positive effects on global cognitive functioning after exercise.

3.3.3.2. Specific neurocognitive advances. When reviewing exercise effects on specific cognitive domains, we found inconsistent results. Domains being assessed included visuospatial/constructional abilities, memory and learning, language, attention/concentration and executive functioning. Not one of these domains consistently improved following exercise. Thus, it seems that while exercise can benefit global cognitive functioning, we cannot yet speculate as to which specific domains may be more prone to change.

\subsubsection{Mediating factors}

3.3.4.1. Diagnosis. While two studies investigated the effects of exercise in a TBI population (Chin et al., 2015; McMillian et al., 2002), ten others focused solely on stroke victims (El-Tamawy et al., 2014; Fang et al., 2003; Kluding et al., 2011; Marzolini et al., 2013; Moore et al., 2015; Nilsson et al., 2001; Ploughman et al., 2008; Pyöriä et al., 2007; Quaney et al., 2009; Studenski et al., 2005). Studies in the latter population sometimes excluded patients who had experienced multiple strokes (Fang et al., 2003; Marzolini et al., 2013; Pyöriä et al., 2007). However, whether or not these patients were included did not appear to effect outcome.

As the results were mixed for both stroke and TBI populations, it would appear that diagnosis is not a determining factor in the ability of exercise to 
influence cognition. However, we certainly need to be cautious about this conclusion, as there were only two TBI studies available.

\subsubsection{Time after injury. Six of the studies investi-} gating the effect of recurring exercise, worked with patients with a mean time after injury of less than one year (Bateman et al., 2001; Fang et al., 2003; McMillian et al., 2002; Nilsson et al., 2001; Pyöriä et al., 2007; Studenski et al., 2005). Two of these studies compare exercise groups (Nilsson et al., 2001 Pyöriä et al., 2007), making it difficult to determine whether additional exercise benefits cognition. The four remaining studies were categorized as level 1 and presented with good quality ratings (Bateman et al., 2001; Fang et al., 2003; McMillian et al., 2002; Studenski et al., 2005). Physiotherapy was compared to relaxation (Bateman et al., 2001), attentional training (McMillian et al., 2002), visits by research staff (Studenski et al., 2005) and no additional intervention above usual care (Fang et al., 2003). None found significant effects in favor of the exercise condition, which makes us conclude that additional exercise within the first year after a brain injury may not lead to enhanced cognitive functioning.

One study did not report the mean duration of illness, but reported a range which surpassed one year post-injury (El-Tamawy et al., 2014). This study, together with five others where the duration of illness was more than one year, found positive effects on cognition (Chin et al., 2015; Kluding et al., 2011; Marzolini et al., 2013; Moore et al., 2015; Quaney et al., 2009). This accumulated evidence of fair to good methodological quality, makes us conclude that after one year, exercise can improve cognition.

\subsubsection{Cognitive impairments at baseline. Unfor-} tunately, only about half of the included studies formulated in/exclusion criteria based on cognitive status (El-Tamawy et al., 2014; Kluding et al., 2011; McMillian et al., 2002; Moore et al., 2015; Ploughman et al., 2008; Quaney et al., 2009; Studenski et al., 2005). The results of these studies were conflicting, with several high quality studies formulating different conclusions. Thus for now, we cannot state that the presence of cognitive problems at baseline is a deciding factor in the effectiveness of exercise interventions.

\subsubsection{Follow-up}

Six RCT's assessed cognition not only immediately after the intervention, but also at follow-up
(Bateman et al., 2001; Fang et al., 2003; McMillian et al., 2002; Nilsson et al., 2001; Quaney et al., 2009; Studenski et al., 2005). Half of these studies have losses to follow-up of twenty or more percent (i.e. 20\% (Studenski et al., 2005), 24.14\% (McMillian et al., 2002) and $83.33 \%$ (Fang et al., 2003)), which poses a serious threat to the validity of their findings (Dettori, 2011). Thus, we will not take them into consideration for our conclusion regarding the long-term effects of exercise. The three remaining publications are of equal methodological quality (i.e. level 1 studies, PEDro ratings of 'good'). One of these studies compares two types of aerobic exercise (Nilsson et al., 2001), again making it difficult to determine whether AT or usual care was accountable for the improvements seen immediately post-intervention and thus also at follow-up. The two remaining studies compared AT to relaxation (Bateman et al., 2001) and stretching (Quaney et al., 2009). Only the latter was able to find a significant difference between groups immediately after the intervention. Eight weeks later the effects had dissipated together with the gains in aerobic fitness. Thus, exercising may not lead to longterm effects on cognition.

\section{Discussion}

With merely 14 relevant studies, there seems to be a void in our current knowledge base, which is more pronounced for TBI patients, rendering only two relevant articles of adequate methodological rigor (Chin et al., 2015; McMillian et al., 2002). These patients are generally younger than stroke victims, which was confirmed by the mean age ranges of the included articles. Knowing that a large group of TBI patients tend to be in or just before the most productive years of their life (Faul, Xu, Wald, \& Coronado, 2010), it is our opinion that this group deserves special attention concerning rehabilitation services. Unfortunately, the physical exercise research for TBI patients is still in the initial stages (Pawlowski, Dixon-Ibarra, \& Driver, 2013).

Apart from the lack of studies in this field, the research that has been done is often methodologically flawed with a combination of small sample groups, high dropout rates, a lack of or ill-designed control groups and a failure to blind assessors, therapists and patients. The studies are also marked by heterogeneity on many levels which is why we did not perform a meta-analysis. We will however formulate tentative answers to our research questions. 


\subsection{Upper and lower limits}

We sought to determine the upper and lower limits of exercise needed to induce cognitive changes. In healthy subjects, even short bouts of exercise have been proven to yield positive effects on cognition (Chang, Labban, Gapin, \& Etnier, 2012; Nanda, Balde, \& Manjunatha, 2013; Yanagisawa et al., 2009). With only one level 2 study of adequate quality available (Ploughman et al., 2008), we are in no position to draw definite conclusions, especially since this study is only of fair methodological quality (i.e. PEDro score: 5/10). In any case, the authors were not able to replicate beneficial effects such as those found in healthy subjects. Knowing that physical therapy sessions in stroke rehabilitation settings are often not of an adequate intensity to elicit cardiovascular treatment effects with patients out of their target heart rate zone for approximately $95 \%$ of the time (MacKay-Lyons \& Makrides, 2002), we question whether a 20-minute walk would have been enough to induce effects on cognition.

Dose-dependent prospective studies on the effect of recurring exercise on cognition have been performed in brain injured animals, with evidence to support both upper and lower limits (MacLellan et al., 2011; Shen et al., 2013). However, this type of research does not yet exist for TBI or stroke patients. None of the included studies compared exercise intensities. We did find that the number of minutes per session and the number of times a patient exercised each weak did not affect outcome. Perhaps because exercising at least three times a week for 30 minutes or more surpasses the lower limit. Furthermore, the evidence suggests that patients need to keep exercising for over four weeks to get results on cognitive function. However, two studies failed to find these positive effects even though the exercise program lasted twelve weeks (Bateman et al., 2001; Studenski et al., 2005). Notably, these studies shared the same outcome measure, namely FIM-cog (Functional Independence Measure, Cognition Subscale). Perhaps this scale is not sensitive enough to pick up subtle changes in cognition as it relies completely on observation, while neuropsychological tests such as the Trail Making Test (TMT) and the Montreal Cognitive Assessment (MoCA) do not. Another possible confounding factor is time postinjury. These particular studies had patient groups with a short time after injury, which may have affected outcome.
We feel it is important to remember that for people with an ABI, a condition often accompanied by motor problems, exercising is not a simple task (Driver, Ede, Dodd, Stevens, \& Warren, 2012; Rimmer, Wang, \& Smith, 2008). Thus, it is imperative to delineate the lower limits needed to achieve results.

\subsection{Type of exercise}

We wanted to learn whether a specific type of exercise is (more) effective at generating cognitive changes. The current research base shows that AT can produce positive effects on cognition, regardless of whether it is performed on a bicycle, on the ground or on a treadmill (Chin et al., 2015; El-Tamawy et al., 2014; Nilsson et al., 2001; Quaney et al., 2009).

The combination of AT and RT also shows promising results. However, only two studies of this type exist and both exhibit methodological shortcomings such as the absence of a control group (Kluding et al., 2011; Marzolini et al., 2013). Notwithstanding the fact that both studies indicate that cognitive improvements can be made, it would be wise to hold off definite conclusions until higher quality studies with control groups have been carried out.

Combined exercise programs are difficult to compare because of the diverse content of the programs. Not surprisingly, the results were mixed (Fang et al., 2003; Moore et al., 2015; Studenski et al., 2005). Only one study of three was able to find a positive effect of exercise on cognition (Moore et al., 2015). This may be attributed either to the fact that this study's exercise regimen lasted several weeks longer or to the difference in duration of illness or to the added benefit of exercising in a group and the social contact that usually ensues.

One particularly interesting finding was that the more traditional physical therapy methods may not challenge a patient enough to induce cognitive changes. In this respect, a comparison study between traditional physical therapy - where the therapist sets goals and chooses methods - and activating physiotherapy - where the patients participate in planning and executing therapy - found higher levels of cognitive functioning in the latter group (Pyöriä et al., 2007). Perhaps, this is because activating therapy can be regarded as a combination of physical and cognitive training. Unfortunately, the activating group received more physiotherapy, which may have been a contributing factor in the difference between groups. 


\subsection{Type of cognitive changes}

Our third goal was to determine which neurocognitive functions could be influenced through physical exercise. In healthy older people, executive function appears to be most susceptible to change (Colcombe $\&$ Kramer, 2003). In our review, the results did not unvaryingly show improvements in executive functioning, nor were there consistent changes in other cognitive domains. These mixed findings may be partly due to the high variability in the measures that were used and the neurocognitive functions they intend to measure.

In general though, the evidence supports the fact that exercise has a positive effect on global cognitive functioning in our population (Chin et al., 2015; ElTamawy et al., 2014; Marzolini et al., 2013; Moore et al., 2015). All but one study - admittedly of good methodological quality - corroborate this statement (Fang et al., 2003). In this particular study, patients were only a maximum of a week after injury and were still receiving usual care. As discussed previously, this may be a reason for the lack of effect on cognition. Another contributing factor may be that, compared to the other studies, the duration of the intervention program was particularly short. Furthermore, the Mini Mental State Examination (MMSE) a screening tool for dementia - was used. This measure may not be well-suited for evaluating changes in cognition (Dong et al., 2010; Nys et al., 2005).

\subsection{Long-term effects}

We wanted to find out whether the effects of exercise were of a transitory or more permanent nature. After accounting for methodological shortcomings, two studies (Bateman et al., 2001; Quaney et al., 2009) - both level 1 and of good quality - were considered for reaching a conclusion. Just one found a significant effect immediately after the intervention. However, these advances were limited to conditional motor learning, and the effects had faded at follow-up. Consequently, the limited research at hand suggests that aerobic training does not yield long-term effects on neurocognitive functioning. Perhaps in order to maintain positive effects in specific areas such as learning, one has to continue exercising. Alternately, a study in mice suggests that lasting changes may result from the combination of physical exercise and cognitive training (Smith et al., 2013). Both hypotheses need to be further assessed in human trials with follow-up evaluations months to years later.

\subsection{Mediating factors}

\subsubsection{Diagnosis}

The mixed findings for both populations would lead one to posit that the type of brain injury has no bearing on the effectiveness of the exercise intervention. However, even though the two TBI studies (Chin et al., 2015; McMillian et al., 2002) point in different directions, we need to bear in mind that the methodologically stronger study indicates that exercising is futile when it comes to improving cognition (McMillian et al., 2002). Nevertheless, before one can conclude that exercise does not help for TBI patients, more research needs to be done accounting for other factors such as time post-injury, which may have been a determining factor in the difference in outcome between these particular studies.

\subsubsection{Time after injury}

One notable finding was that the studies evaluating the effect of recurring exercise were all able to find a significant effect on cognition in patients whose brain injury occurred over a year before (Chin et al., 2015; Kluding et al., 2011; Marzolini et al., 2013; Moore et al., 2015; Quaney et al., 2009). In contrast, the studies with patients whose mean duration of illness was less than one year were unable to find these exercise effects (Bateman et al., 2001; Fang et al., 2003; McMillian et al., 2002; Studenski et al., 2005). Within one year post-injury, patients are still in the acute to post-acute stage of a brain injury (Spreen, Risser, \& Edgell, 1995). Therefore, it is not surprising that in five of these six studies, patients were still receiving usual care (Bateman et al., 2001; Fang et al., 2003; Nilsson et al., 2001; Pyöriä et al., 2007; Studenski et al., 2005). They started the exercise regimen sometimes just days after injury. Thus, one would also expect progress with usual care alone or even without usual care (Cramer, 2008). Perhaps when patients are still receiving usual care, which can be quite intensive and often includes some form of physiotherapy, additional physical exercise yields no added benefit. This would mean that a specific time period exists in which physical exercise would be most beneficial. More research needs to be done to determine this specific time period.

\subsubsection{Cognitive impairments at baseline}

Based on the present literature, one could - perhaps prematurely - conclude that pre-intervention cognitive dysfunctions do not determine the effectiveness of an exercise intervention. However, we found 
it difficult to determine whether or not these symptoms were present. First of all, only about half of the studies used cognitive parameters for in/exclusion criteria (El-Tamawy et al., 2014; Kluding et al., 2011; McMillian et al., 2002; Moore et al., 2015; Ploughman et al., 2008; Quaney et al., 2009; Studenski et al., 2005). Secondly, five of the seven trials that did have cognitive in/exclusion criteria used cut-off scores on the MMSE (Kluding et al., 2011; Moore et al., 2015; Ploughman et al., 2008; Quaney et al., 2009; Studenski et al., 2005), a measure that has been shown to underestimate cognitive impairments after stroke (Nys et al., 2005; Pendlebury, Cuthbertson, Welch, Mehta, \& Rothwell, 2010). Thus, future studies would do well to take this criterium into consideration and use tests or cut-off scores specifically designed for this population. Only then can we conclude whether or not it would influence outcome.

\subsection{Study limitations}

There are several limitations to this review. For one, the language of the publications was restricted to English and we only used data from PubMed and ScienceDirect, thus possibly omitting relevant articles. Secondly, given the limited research that was available, we chose to include both controlled and single group designs. Quasi-experimental designs pose a greater threat to internal validity (Coryn, \& Hobson, 2011). Consequently, the results of the studies used in this review may have been confounded and with them perhaps our conclusions. Thirdly, the search terms may not have been inclusive enough, as five of the fourteen included studies were not found through the initial database search.

\subsection{Suggestions for further research}

Future research can pursue several avenues of investigation. First, it is important to firmly establish the link between structural and neurocognitive changes due to exercise in our population. Secondly, several researchers have already started substantiating the relationship between improvements in aerobic fitness and enhanced cognition (Chin et al., 2015; Kluding et al., 2011; Marzolini et al., 2013). It would be interesting to assess whether a similar link exists between improvements in strength indices and cognitive function. Thirdly, indirect evidence suggests a benefit of adding cognitive training to a physical exercise regimen. Animal research has shown that the combination of both forms of training exceeds the benefits of either treatment alone (Langdon \& Corbett, 2012), possibly with long-term effects on cognition (Smith et al., 2013). Also, a recent meta-analysis showed that this combination can be beneficial for both healthy subjects and people with cognitive impairments (Law, Barnett, Yau, \& Gray, 2014). Furthermore, there is the superiority of activating therapy over traditional therapy (Pyöriä et al., 2007) and the beneficial effects of combining exercise with recreation which often includes the learning of new skills (Rand et al., 2010). This evidence, though circumstantial, certainly warrants further investigation. Fourthly, future research could focus on determining whether non-traumatic brain injury patients and TBI patients respond differently to exercise. We attempted to compare studies with these populations, but due to the limited number of TBI studies, we were unable to draw conclusions. Lastly, we recommend further investigation of cognitive dysfunctions at baseline and the role of time after injury. We already raised the issue that, in addition to usual care, exercising within a year after injury may not have added value. However, more worrisome than lost time and energy is the fact that animal studies suggest that exercising too soon after a TBI may be damaging instead of beneficial (Griesbach, 2011).

\section{Conclusion}

Acquired brain injuries pose a huge and increasing burden on patients, their environment and our society. Thus, investigating and developing new ways to treat these neurologic conditions should be a priority. Physical exercise appears to be a safe, non-invasive rehabilitation method to alleviate cognitive problems, especially in stroke patients. Unfortunately, there is a paucity of research, and methodological concerns exist regarding the studies that are available. As some of our questions remain unanswered, there is a need for well-designed studies. We can however, state that the majority of studies reveal a positive effect of recurring physical exercise on global cognitive functioning even though specific areas more prone to improvement could not be identified. Both AT and the combination of AT and RT appear to be effective at inducing these changes, although long-term changes have not yet been found. Ideally, an exercise program should last over four weeks, with patients exercising at least three times a week for thirty minutes. We also recommend that patients be actively involved in formulating goals. This not only raises motivation 
but may have a positive effect on cognition in itself. Finally, additional exercise may be most beneficial in the chronic stages of a brain injury, as studies with patient groups less than a year after injury were generally not able to find a beneficial effect over usual care.

\section{Acknowledgments}

We would like to thank physiotherapist Niels Habraken for his valuable contributions to this work.

\section{Conflict of interest}

The authors have no conflict of interest to report.

\section{References}

Ambroginia, P., Lattanzia, D., Ciuffolia, S., Bettia, M., Fanellib, M., \& Cuppinia, R. (2013). Physical exercise and environment exploration affect synaptogenesis in adult-generated neurons in the rat dentate gyrus: Possible role of BDNF. Brain Research, 1534, 1-12.

Aubut, J. A., Marshall, S., Bayley, M., \& Teasell, R. W. (2013). A comparison of the PEDro and Downs and Black quality assessment tools using the acquired brain injury intervention literature. NeuroRehabilitation, 32(1), 95-102.

Bateman, A., Culpan, F. J., Pickering, A. D., Powell, J. H., Scott, O. M., \& Greenwood, R. J. (2001). The effect of aerobic training on rehabilitation outcomes after recent severe brain injury: A randomized controlled evaluation. Archives of Physical Medicine and Rehabilitation, 82(2), 174-182.

Benjamin, D. R., van de Water, A. T. M., \& Peiris, C. L. (2014). Effects of exercise on diastasis of the rectus abdominis muscle in the antenatal and postnatal periods: A systematic review. Physiotherapy, 100(1), 1-8.

Bhogal, S. K., Teasell, R. W., \& Foley, N. C. (2005). The PEDro scale provides a more comprehensive measure of methodological quality than the Jadad Scale in stroke rehabilitation literature. Journal of Clinical Epidemiology, 58(7), 668-673.

Casla, S., Hojman, P., Márquez-Rodas, I., López-Tarruella, S., Jerez, Y., Barakat, R., \& Martín, M. (2014). Running away from side effects: Physical exercise as a complementary intervention for breast cancer patients. Clinical and Translational Oncology, 17(3), 180-196.

Centers for Disease Control and Prevention (2014). Report to Congress on Traumatic Brain Injury in the United States: Epidemiology and Rehabilitation. National Center for Injury Prevention and Control; Division of Unintentional Injury Prevention. Atlanta, GA.

Chang, Y. K., Labban, J. D., Gapin, J. I., \& Etnier, J. L. (2012). The effects of acute exercise on cognitive performance: A metaanalysis. Brain Research, 1453, 87-101.

Chin, L. M., Keyser, R. E., Dsurney, J., \& Chan, L. (2015). Improved cognitive performance following aerobic exercise training in people with traumatic brain injury. Archives of Physical Medicine and Rehabilitation, 96(4), 754-759.
Cicerone, K. D., \& Azulay, J. (2007). Perceived self-efficacy and life satisfaction after traumatic brain injury. The Journal of Head Trauma Rehabilitation, 22(5), 257-266.

Colcombe, S., \& Kramer, A. F. (2003). Fitness effects on the cognitive function of older adults: A meta-analytic study. Psychological Science, 14(2), 125-130.

Coryn, C. L. S., \& Hobson, K. A. (2011). Using nonequivalent dependent variables to reduce internal validity threats in quasiexperiments: Rationale, history, and examples from practice. In S. Mathison (Ed.), Really new directions in evaluation: Young evaluators' perspectives. New Directions for Evaluation, 131, 31-39.

Cramer, S. C. (2008). Repairing the human brain after stroke: I. mechanisms of spontaneous recovery. Annals of Neurology, 63(3), 272-287.

Cumming, T. B., Tyedin, K., Churilov, L., Morris, M. E., \& Bernhardt, J. (2012). The effect of physical activity on cognitive function after stroke: A systematic review. International Psychogeriatrics, 24(4), 557-567.

Cumming, T. B., Brodtmann, A., Darby, D., \& Bernhardt, J. (2014). The importance of cognition to quality of life after stroke. Journal of Psychosomatic, 77(5), 374-379.

Dettori, J. R. (2011). Loss to follow-up. Evidence-Based SpineCare Journal, 2(1), 7-10.

Devine, J. M., \& Zafonte, R. D. (2009). Physical exercise and cognitive recovery in acquired brain injury: A review of the literature. The American Academy of Physical Medicine and Rehabilitation, 1(6), 560-575.

Dong, Y., Sharma, V. K., Chan, B. P.-L., Venketasubramanian, N., Teoh, H. L., Seet, R. C., .. \& \& Chen, C. (2010). The Montreal Cognitive Assessment (MoCA) is superior to the Mini-Mental State Examination (MMSE) for the detection of vascular cognitive impairment after acute stroke. Journal of the Neurological Sciences, 299(1-2), 15-18.

Downs, S. H., \& Black, N. (1998). The feasibility of creating a checklist for the assessment of the methodological quality both of randomised and non-randomised studies of health care interventions. Journal of Epidemiology \& Community Health, 52(6), 377-384.

Driver, S., Ede, A., Dodd, Z., Stevens, L., \& Warren, A. M. (2012). What barriers to physical activity do individuals with a recent brain injury face? Disability and Health Journal, 5(2), 117125.

Duman, R. S. (2005). Neurotrophic factors and regulation of mood: Role of exercise, diet and metabolism. Neurobiology of Aging, 26(Suppl. 1), 88-93.

Durstine, J. L., Gordon, B., Wang, Z., \& Luo, X. (2013). Chronic disease and the link to physical activity. Journal of Sport and Health Science, 2(1), 3-11.

El-Tamawy, M. S., Abd-Allah, F., Ahmed, S. M., Darwish, M. H., \& Khalifa, H. A. (2014). Aerobic exercises enhance cognitive functions and brain derived neurotrophic factor in ischemic stroke patients. NeuroRehabilitation, 34(1), 209-213.

Eng, J. J., Teasell, R., Miller, W. C., Wolfe, D. L., Townson, A. F., Aubut, J.-A., . . \& T The SCRIRE Research Team (2007). Spinal cord injury rehabilitation evidence: Methods of the SCIRE systematic review. Top Spinal Cord Injury Rehabilitation, 13(1), $1-10$.

Erickson, K. I., Leckie, R. L., \& Weinstein, A. M. (2014). Physical activity, fitness, and gray matter volume. Neurobiology of Aging, 35(Suppl. 2), 20-28. 
Fang, Y., Chen, X., Li, H., Lin, J., Huang, R., \& Zeng, I. (2003). A study on additional early physiotherapy after stroke and factors affecting functional recovery. Clinical Rehabilitation, 17(6), 608-617.

Faul, M., Xu, L., Wald, M. M., \& Coronado, V. G. (2010). Traumatic Brain Injury in the United States: Emergency Department Visits, Hospitalizations and Deaths 2002-2006. Atlanta (GA): Centers for Disease Control and Prevention, National Center for Injury Prevention and Control.

Fogelman D., \& Zafonte, R. (2012). Exercise to enhance neurocognitive function after traumatic brain injury. American Academy of Physical Medicine and Rehabilitation, 4(11), 908-913.

Foley, N. C., Bhogal, S. K., Teasell, R. W., Bureau, Y., \& Speechley, M. R. (2006). Estimates of quality and reliability with the physiotherapy evidence-based database scale to assess the methodology of randomized controlled trials of pharmacological and nonpharmacological interventions. Physical Therapy, 86(6), 817-824.

Grealy, M. A., Johnson, D. A., \& Rushton, S. K. (1999). Improving cognitive function after brain injury: The use of exercise and virtual reality. Archives of Physical Medicine and Rehabilitation, 80(6), 661-667.

Griesbach, G. S. (2011). Exercise after traumatic brain injury: Is it a double-edged sword? American Academy of Physical Medicine and Rehabilitation, 3(6 Suppl. 1), 64-72.

Hebb, D. O. (1949). The Organization of Behavior. A Neurophysiological Theory. New York: Wiley.

Heyn, P., Abreu, B. C., \& Ottenbacher, K. J. (2004). The effects of exercise training on elderly persons with cognitive impairment and dementia: A meta-analysis. The Archives of Physical Medicine and Rehabilitation, 85(10), 1694-1704.

Hooper, P., Jutai, J. W., Strong, G., \& Russsel-Minda, E. (2008). Age-related macular degeneration and low-vision rehabilitation: A systematic review. Canadian Journal of Ophthalmology, 43(2), 180-187.

Hötting, K., \& Röder, B. (2013). Beneficial effects of physical exercise on neuroplasticity and cognition. Neuroscience and Biobehavioral Reviews, 37(9, Pt. B), 2243-2257.

Jadad, A. R., Moore, R. A., Carroll, D., Jenkinson, C., Reynolds, D. J., Gavaghan, D. J., \& McQuay, H. J. (1996). Assessing the quality of reports of randomized clinical trials: Is blinding necessary? Controlled Clinical Trials, 17(1), 1-12.

Kim, J., Choi, W. J., \& Jeong, S. H. (2013). The effects of physical activity on breast cancer survivors after diagnosis. Journal of Cancer Prevention, 18(3), 193-200.

Kluding, P. M., Tseng, B. Y., \& Billinger, S. A. (2011). Exercise and executive function in individuals with chronic stroke: A pilot study. Journal of Neurological Physical Therapy, 35(1), 11-17.

Langdon, K., \& Corbett, D. (2012). Improved working memory following novel combinations of physical and cognitive activity. Neurorehabilitation and Neural Repair, 26(5), 523-532.

Law, L. L., Barnett, F., Yau, M. K., \& Gray, M. A. (2014). Effects of combined cognitive and exercise interventions on cognition in older adults with and without cognitive impairment: A systematic review. Ageing Research Reviews, 15, 62-75.

Lee, I.-M., \& Skerrett, P. J. (2001). Physical activity and all-cause mortality: What is the dose-response relation? Official Journal of the American College of Sports Medicine, 33(Suppl. 6), 459471.

Liberati, A., Altman, D. G.,Tetzlaff, J., Mulrow, C., Gøtzsche, P. C., Ioannidis J. P. A., · \& Moher, D. (2009). The PRISMA statement for reporting systematic reviews and meta-analyses of studies that evaluate health care interventions: Explanation and elaboration. Public Library of Science Medicine, 6(7), e1000100.

MacKay-Lyons, M. J., \& Makrides, L. (2002). Cardiovascular stress during a contemporary stroke rehabilitation program: Is the intensity adequate to induce a training effect? Archives of Physical Medicine and Rehabilitation, 83(10), 1378-1383

MacLellan, C. L., Keough, M. B., Granter-Button, S., Chernenko, G. A., Butt, S., \& Corbett, D. (2011). A critical threshold of rehabilitation involving brain-derived neurotrophic factor is required for poststroke recovery. Neurorehabilitation and Neural Repair, 25(8), 740-748.

Maher, C. G., Sherrington, C., Herbert, R. D., Moseley, A. M., \& Elkins, M. (2003). Reliability of the PEDro scale for rating quality of randomized controlled trials. Physical Therapy, 83(8), 713-721.

Marzolini, S., Oh, P., McIlroy, W., \& Brooks, D. (2013). The effects of an aerobic and resistance exercise training program on cognition following stroke. Neurorehabilitation and Neural Repair, 27(5), 392-402.

McDonnell, M. N., Smith, A. E., \& Mackintosh, S. F. (2011). Aerobic exercise to improve cognitive function in adults with neurological disorders: A systematic review. The Archives of Physical Medicine and Rehabilitation, 92(7), 1044-1052.

McMillian, T., Robertson, I. H., Brock, D., \& Chorlton, L. (2002). Brief mindfulness training for attentional problems after traumatic brain injury: A randomised control treatment trial. Neuropsychological Rehabilitation, 12(2), 117-125.

Meeusen, R. (2005). Exercise and the brain: Insight in new therapeutic modalities. Annals of Transplantation: Quarterly of the Polish Transplantation Society, 10(4), 49-51.

Moore, S. A., Hallsworth, K., Jakovljevic, D. G., Blamire, A. M., Jiabao, H., Ford, G. A., .. \&\& Trenell, M. I. (2015). Effects of community exercise therapy on metabolic, brain, physical, and cognitive function following stroke: A randomized controlled pilot trial. Neurorehabilitation and Neural Repair, 29(7), 623635.

Mozaffarian, D., Benjamin, E., Go A., Arnett, D., Blaha, M., Cushman, M., ...\& Turner, M. (2015). Heart disease and stroke statistics-2015 update: A report from the American Heart Association. Circulation, 2015, e29-e322.

Nanda, B., Balde, J., \& Manjunatha, S. (2013). The acute effects of a single bout of moderate-intensity aerobic exercise on cognitive functions in healthy adult males. Journal of Clinical and Diagnostic Research, 7(9), 1883-1885.

Nilsson, L., Carlsson, J., Danielsson, A., Fugl-Meyer, A., Hellström, K., Kristensen, L., ...\& Grimby, G. (2001). Walking training of patients with hemiparesis at an early stage after stroke: A comparison of walking training on a treadmill with body weight support and walking training on the ground. Clinical Rehabilitation, 15(5), 515-527.

Nys, G. M. S., van Zandvoort, M. J. E., de Kort, P. L. M., Jansen, B. P. W., Kappelle, L. J., \& de Haan, E. H. F. (2005). Restrictions of the Mini-Mental State Examination in acute stroke. Archives of Clinical Neuropsychology, 20(5), 623-629.

Ogoh, Z., \& Ainslie, P. N. (2009). Cerebral blood flow during exercise: Mechanisms of regulation. Journal of Applied Physiology, 107(5), 1370-1380.

Olesen, J., Gustavsson, A., Svensson, M., Wittchen, H.-U., \& Jönsson, B on behalf of the CDBE2010 study group and the 
European Brain Council (2012). The economic cost of brain disorders in Europe. European Journal of Neurology, 19(1), 155-162.

Olivo, S. A., Macedo, L. G., Gadotti, I. C., Fuentes, J., Stanton, T., \& Magee, D. J. (2008). Scales to assess the quality of randomized controlled trials: A systematic review. Physical Therapy, $88(2), 156-175$

Pawlowski, J., Dixon-Ibarra, A., \& Driver, S. (2013). Review of the status of physical activity research for individuals with traumatic brain injury. Archives of Physical Medicine and Rehabilitation, 94(6), 1184-1189.

Pendlebury, S. T., Cuthbertson, F. C., Welch, S. J. V., Mehta, Z., \& Rothwell, P. M. (2010). Underestimation of cognitive impairment by mini-mental state examination versus the montreal cognitive assessment in patients with transient ischemic attack and stroke. A population-based study. Stroke, 41(6), 1290-1293.

Ploughman, M., McCarthy, J., Bossé, M., Sullivan, H. J., \& Corbett, D. (2008). Does treadmill exercise improve performance of cognitive or upper-extremity tasks in people with chronic stroke? A randomized cross-over trial. Archives of Physical Medicine and Rehabilitation, 89(11), 2041-2047.

Prigatano, G. P., Fordyce, D. J., Zeiner, H. K., Roueche, J. R., Pepping, M., \& Wood, B. C. (1986). Neuropsychological rehabilitation after brain injury. Baltimore, MD: Johns Hopkins University Press.

Pyöriä, O., Talvitie, U., Nyrkkö, H., Kautiainen, H., Pohjolainen, T., \& Kasper, V. (2007). The effect of two physiotherapy approaches on physical and cognitive functions and independent coping at home in stroke rehabilitation. A preliminary follow-up study. Disability and Rehabilitation, 29(6), 503-511.

Quaney, B. M., Boyd, L. A., McDowd, J. M., Zahner, L. H., He, J., Mayo, M. S., \& Macko, R. F. (2009). Aerobic exercise improves cognition and motor function poststroke. Neurorehabilitation and Neural Repair, 23(9), 879-885.

Rand, D., Eng, J. J., Liu-Ambrose, \& T., Tawashy, A. E. (2010). Feasibility of a 6-month exercise and recreation program to improve executive functioning and memory in individuals with chronic stroke. Neurorehabilitation and Neural Repair, 24(8), 722-729.

Redila, V. A., \& Christie, B. R. (2006). Exercise-induced changes in dendritic structure and complexity in the adult hippocampal dentate gyrus. Neuroscience, 137(4), 1299-1307.

Rimmer, J. H., Wang, E., \& Smith, D. (2008). Barriers associated with exercise and community access for individuals with stroke. Journal of Rehabilitation Research \& Development, 45(2), 315-322.

Saunders, L. D., Soomro, G. M., Buckingham, J., Jamtvedt, G., \& Raina, P. (2003). Assessing the methodological quality of nonrandomized intervention studies. Western Journal of Nursing Research, 25(2), 223-237.

Schmidt, W., Endres, M., Dimeo, F., \& Jungehulsing, G. (2013). Train the vessel, gain the brain: Physical activity and vessel function and the impact on stroke prevention and outcome in cerebrovascular disease. Cerebrovascular Diseases, 35(4), 303-312.

Shen, X., Li, A., Zhang, Y., Dong, X., Shan, T., Wu, Y., ... \& Hu, Y. (2013). The effect of different intensities of treadmill exercise on cognitive function deficit following a severe controlled cortical impact in rats. International Journal of Molecular Sciences, 14(11), 21598-21612.

Smith, A. M., Spiegler, K. M., Sauce, B., Wass, C. D., Sturzoiu, T., \& Matzel, L. D. (2013). Voluntary aerobic exercise increases the cognitive enhancing effects of working memory training. Behavioral Brain Research, 256, 625-635.

Spreen, O., Risser, A.H., \& Edgell, D. (1995). Developmental neuropsychology. New York, NY: Oxford University Press.

Studenski, S., Duncan, P. W., Perera, S., Reker, D., Lai, S. M., \& Richards, L. (2005). Daily functioning and quality of life in a randomized controlled trial of therapeutic exercise for subacute stroke survivors. Stroke, 36(8), 1764-1770.

Szuhany, K. L., Bugatti, M., \& Otto, M. W. (2015). A meta-analytic review of the effects of exercise on brain-derived neurotrophic factor. Journal of Psychiatric Research, 60, 56-64.

van Praag, H., Christie, B. R., Sejnowski, T. J., \& Gage, F. H. (1999). Running enhances neurogenesis, learning, and long-term potentiation in mice. Proceedings of the National Academy of Sciences, 96(23), 13427-13431.

van Praag, H. (2008). Neurogenesis and exercise: Past and future directions. Neuromolecular Medicine, 10(2), 128-140.

Yanagisawa, H., Dan, I., Tsuzuki, D., Kato, M., Okamoto, M. Kyutoku, Y., \& Soya, H. (2009). Acute moderate exercise elicits increased dorsolateral prefrontal activation and improves cognitive performance with Stroop test. NeuroImage, 40(4), $1702-1710$

Yang, P.-Y., Ho, K.-H., Chen, H.-C., \& Chien, M.-Y. (2012). Exercise training improves sleep quality in middle-aged and older adults with sleep problems: A systematic review. Journal of Physiotherapy, 58(3), 157-163. 